\title{
MicroRNA-200c-3p Functions as a Tumor \\ Suppressor in Kidney Renal Clear Cell Carcinoma by Targeting Vascular Endothelial Growth Factor A
}

\section{Yanmin Li}

First Affiliated Hospital of Guangzhou Medical University

Hui Xu

First Affiliated Hospital of Gannan Medical University

Qianghai Wen

First Affiliated Hospital of Gannan Medical University

Xiaoqi Yan

First Affiliated Hospital of Gannan Medical University

Gang xu ( $\sim$ x15970729228@163.com )

First Affiliated Hospital of Gannan Medical University

\section{Research}

Keywords: miR-200c-3p, VEGFA, KIRC,

Posted Date: October 11th, 2021

DOl: https://doi.org/10.21203/rs.3.rs-952406/v1

License: (9) This work is licensed under a Creative Commons Attribution 4.0 International License. Read Full License 


\section{Abstract}

Background: MicroRNA(miR)-200c-3p is a tumor suppressor that helps inhibit the progression of various types of cancer. However, its role in kidney renal clear cell carcinoma (KRIC) is unknown. The purpose of this study was to explore the biological function and regulatory mechanism of miR-200c-3p in the development of KRIC.

Methods: The relative levels of miR-200c-3p and vascular endothelial growth factor A (VEGFA) in KIRC tissues and cells were determined using the qRT-PCR technique. Transwell and wound healing assay methods were used to understand the effect of miR-200c-3p on the migration and invasion of 786-0 and Caki-1 cells. The ability of miR-200c-3p to target VEGFA was determined using the Dual-Luciferase reporter assay system

Results: MiR-200c-3p was downregulated in KIRC tissues and cell lines. The overexpression of miR-200c$3 p$ attenuated the migratory capacities of $786-0$ and Caki- 1 cells. The migratory ability increased under conditions of miR-200c-3p knockdown. Analysis of the results obtained using the wound healing assay revealed that overexpression of miR-200c-3p reduced the migratory abilities of Caki- 1 and 786-0 cells, and inhibition of miR-200c-3p accelerated the process of wound closure. Analysis of the results obtained from screening tests and by conducting the Dual-Luciferase reporter assay revealed that VEGFA was the direct target of miR-200c-3p. The VEGFA mRNA level was low in the 786-0 and Caki-1 cells under conditions of overexpressed miR-200c-3p. High levels of VEGFA mRNA were observed in the cells post miR-200c-3p knockdon.

Conclusions: The tumor suppression ability of miR-200c-3p in KIRC can be achieved by regulating the expression of VEGFA.

\section{Introduction}

Renal cell carcinoma (RCC) is one of the most common types of kidney cancers. RCC accounts for approximately $39 \%$ of all cancer cases in adults [1]. Kidney renal clear cell carcinoma (KIRC) is one of the highest incidence subtypes [2]. The tumors are prone to metastasis and KIRC has a poor prognosis [3]. It is challenging to treat metastatic KIRC and effective treatment methods are still being researched. The KIRC transfer mechanism has attracted the attention of researchers. The fact that KIRC is rarely characterized by early symptoms makes the diagnosis of KIRC difficult[4]. Therefore, exploring the molecular elements involved in the regulation of RCC progression can potentially help in the clinical diagnosis of KIRC or the process of targeted intervention.

MiRNAs and small non-coding RNAs (19 to 24 nucleotides long) play important roles in mRNA degradation and transformation inhibition [5-7]. MiRNA regulates tumor migration, invasion, and growth and influences cancer progression [8-10]. MiR-200c-3p is a member of miR-200 family and is also a cancer-related miRNA. Analysis of literature reports revealed that miR-200c-3p plays an important role in regulating various human cancers. It influences the proliferation and metastasis of tumor cells (causing 
colorectal cancer) and glial mother cells [11-12]. However, little is known about the biological function of miR-200c-3p.

An analysis of the data present in the Gene Expression Omnibus (GEO) database GSE95384 revealed that the level of miR-200c-3p expression in the KIRC tissue was significantly lower than the level of expression in the adjacent normal tissues. We also observed that the overexpression of miR-200c-3p could reduce the level of VEGFA and inhibit the migration of the KIRC cells. VEGFA is a member of the PDGF/VEGF growth factor family [13]. It influences tumor angiogenesis and affects tumor growth, metastasis, and survival [14]. Analysis of the obtained data reveals that miR-200c-3p is a tumor suppressor [15-16]. The tumor suppression ability in KIRC can be achieved by targeting VEGFA. KIRC can be potentially treated by following the processes of miR-200c-3p therapy.

\section{Materials And Methods \\ Clinical samples}

The Ethics Committee of the First Affiliated Hospital of Gannan Medical University (Ganzhou, China) approved our study. The experiments in this study were conducted in accordance with the approved guidelines and norms. All the patients enrolled signed informed consent forms. Twenty-six pairs of KIRC tissues and matched adjacent tissues were obtained from the enrolled patients (male, $n=15$; female, $\mathrm{n}=11$ ) undergoing biopsy before being subjected to chemotherapeutics. The ages of the patients were in the range of $35-79$ years ( $\geq 60, n=12 ;<60, n=14$; mean age, $47.88 \pm 12.26$ years). After the specimens were washed with normal saline and the bloodstains were cleaned, they were immediately put into liquid nitrogen and frozen.

\section{Cells and cell culture}

The HK-2, ACHN, A498, Caki-1, and 786-0 cell lines were purchased from the Institute of Biochemistry and Cell Biology of the Chinese Academy of Sciences (Shanghai, China). 786-0 and Caki-1 cells were cultured in a DMEM/F12 medium (Life Technologies, NY) containing 10\% fetal bovine serum (Gibco, NY) and streptomycin/penicillin ( $1 \%$, Gibco, CA). All cells were cultured at $37{ }^{\circ} \mathrm{C}$ in the presence of $5 \% \mathrm{CO}_{2}$.

\section{RNA purifification and qRT-PCR}

The total RNAs from tissue samples (approximately $3.0 \mathrm{~g}$ ) or cells $\left(1 \times 10^{7}\right)$ or cells in Trizol (Invitrogen, United States) were extracted. The ultraviolet spectrophotometry technique was used to determine the concentration and purity of total RNA at $260 \mathrm{~nm}$ and $280 \mathrm{~nm}$. A reverse transcription kit (Thermo Fisher Scientific, Inc.) was used to convert RNA to cDNA. The protocol outlined by the manufacturer was followed to conduct the experiments. The qPCR technique was used to study the samples using SYBR Green Master (TsingKe, China). GAPDH served as the endogenous control of VEGFA. 
Table 1

Primer Sequences for qRT-PCR

\begin{tabular}{|ll|}
\hline Gene & Primer sequence \\
\hline miR200c3p stemloop & 5'GTCGTATCCAGTGCAGGGTCCGAGGTATTCGCACTGGATACGACTCCATC3' \\
\hline miR200c3p & Forward :5'CGCGTAATACTGCCGGGTAAT3' \\
\cline { 2 - 2 } VEGFA & Reverse: 5'AGTGCAGGGTCCGAGGTATT3' \\
& Forward: 5'GCATTGGAGCCTTGCCTT3' \\
\hline GAPDH & Forward: 5'GCACCGTCAAGGCTGAGAAC3 \\
\hline & Reverse: 5'TGGTGAAGACGCCAGTGGA3' \\
\hline
\end{tabular}

\section{Western blotting}

Proteins obtained from cultured cells were subjected to electrophoresis, following which they were transferred on polyvinylidene difluoride membranes. Subsequently, the samples were sealed using $5 \%$ skimmed milk (time: $2 \mathrm{~h}$ ) at room temperature. Following this, the protein was incubated with the specific primary antibody. The sample was refrigerated overnight at $4{ }^{\circ} \mathrm{C}$. Following incubation, the sample was washed three times with PBST. Each washing cycle lasted $10 \mathrm{~min}$. Post washing, the samples were incubated with the secondary antibody (Goat Anti-Rabbit IgG-HRP, 1:5,000, Abmart). Rabbit anti-VEGFA (1:1,000, Abcam) and GAPDH (1:5,000, Abmart) antibodies were used for the experiments.

\section{Transwell assay and wound healing experiment}

Following cell counting, migration analyses experiments were conducted. The transwell co-culture system consisting of a transwell plate (12-well; Corning, MA, United States) and transwell inserts (pore size: 8 $\mathrm{mm}$; Costar, NY, United States) were used for the experiments. $786-0$ or Caki- 1 cells, seeded at $5 \times 10^{4}$ cells per well, were inoculated using transwell inserts (pore size: $8 \mathrm{~mm}$ ). The medium $(200 \mu \mathrm{L})$ devoid of FBS was added to the chamber. The medium $(800 \mu \mathrm{L})$ containing FBS $(10 \%)$ was added to the lower chamber. The samples were incubated at $37^{\circ} \mathrm{C}$ for $24 \mathrm{~h}$, following which the cells were passed through the membrane. The samples were fixed with paraformaldehyde $(4 \%)$ and stained with crystal violet $(0.1 \%)$ for $15 \mathrm{~min}$. The treated samples were then washed several times with distilled water. Finally, the cells on the membrane were observed using a microscope (Nikon, Japan), and the five random visual fields of each hole were photographed and counted.

For the wound healing experiment, following cell counting, the cells were inoculated in a six-hole plate and were scratched using a sterile pipette $(200 \mu \mathrm{L})$. The cells were incubated and grown to a concentration of $90 \%$. Wound closure was observed, and the images were recorded at 0 and $24 \mathrm{~h}$ using a microscope.

\section{Luciferase reporter assay}


The possible binding sites between miRNA-200c-3p and VEGFA in 3'UTR were obtained from the TargetScan 1 database. Lipofectamine 2000 was used to study the overexpression of miR-200c-3p or NC. The data were compared with the data obtained for the cells co-transfected with vectors consisting of wild-type (wt) and mutated (mut) 3'UTR sequence of VEGFA. After $48 \mathrm{~h}$ of transfection, the cells were collected. The double Luciferase Assay System (Promega, Madison, WI, United States) was used to detect the luciferase activities of the co-transfected cells.

\section{Statistical analysis}

All data were expressed as mean \pm standard deviation (SD). The results obtained from the unpaired Student's t-test were used to understand the differences between the two groups. The differences between the VEGFA and miR-200c-3p mRNA expressions in matched tissues were statistically analyzed by conducting paired t-tests. The statistical analyses were performed using GraphPad Prism 9. $P<0.05$ was considered to indicate a statistically significant difference.

\section{Results}

\section{The expression miR-200c-3p was down-regulated in the KIRC tissues andcells}

LIMMA (R package) [17] was used to analyze the GEO database (GSE95384) [18] to detect the expression of miRNAs in the KIRC tissues and cells. The volcano plot revealed the miRNA expression levels in KIRC and adjacent normal tissues (the GSE95384 database was taken into consideration) (Fig. 1A). The heap map showed the down-regulated microRNAs. The color white indicates down-regulation, and the color red indicates up-regulation (Fig. 1B). Analysis of GSE95384 revealed that miR-200c-3p was the most downregulated miRNA. Therefore, we chose miR-200c-3p as the research target. As shown in Fig. 1C, according to the data in GSE95384, the level of expression of miR-200-3p in KIRC tissues was significantly lower than the level of expression in normal tssues. The qPCR technique was used to detect the expression of miR-200c-3p in 32 pairs of tumor tissues and adjacent tissues. The results revealed that miR-200c-3p was significantly down-regulated in tumor tissues (as compared to the adjacent tissues) (Fig. 1C, $P<0.05)$. The level of expression of miR-200c-3p in KIRC cells was lower than the level of expression of miR-200c-3p in HK-2 cells (Fig. 1D, $P<0.05$ ).

\section{MiR-200c-3p suppresses KIRC cells migration, invasion, nd EMT}

Caki-1 and 786-0 cells were used for the in vitro experiments. Wound healing and migration assays were used to determine the extent of migration and invasion. As shown in Figs. 2A-E, the overexpression of miR-200c-3p significantly suppressed cell migration and invasion in the Caki- 1 and 786-0 cells. The inhibition of miR-200c-3p resulted in an increase in the extent of cell migration and suppression. The migration rate observed in the cells containing overexpressed miR-200c-3p was significantly lower than the migration rate observed in the control group $(P<0.01$; Figs. $2 \mathrm{~B}, \mathrm{D}$ and $\mathrm{E})$. 
The results obtained from the wound healing assay revealed that the migratory abilities observed for the Caki-1 and 786-0 cells were reduced under conditions of overexpression. It was also observed that the inhibition of miR-200c-3p accelerated the process of wound closure. The percentage of wound healing in the cells characterized by overexpressed miR-200c-3p was significantly higher than the percentage observed in the control group. The percentage of wound healing in the miR-200c-3p inhibition group was significantly lower than that observed in the control group. These results suggest that miR-200c-3p may play a vital role in regulating the migration and EMT of KIRC cells.

\section{Bioinformatics analysis for the search of the target genes of miR200c-3p}

We used TargetScan, miRDB, Starbase, and GEO as tools to predict the potential targets of miR-200c-3p (Fig. 3A) to understand the working mechanism of miR-200c-3p during the occurrence and development of KIRC. Of all the candidate genes, VEGFA was paid special attention because It plays an extremely important role in regulating the occurrence and development of tumor angiogenesis, and ultimately promotes tumor progression.

VEGFA expression in 32 paired KIRC and adjacent tissues was determined using the qRT-PCR technique. The VEGFA levels in KIRC tissues were significantly higher than the VEGFA levels in the corresponding patient's tumor tissues $(P<0.05$, Fig. 3B). These results were also confirmed by the data obtained from the GEPIA website (Figs. 3D and G). As showed in Figs. 3E-F, VEGFA is expressed in various human tumors. The maximum expression was observed in the case of KIRC. The level of expression was higher in patients suffering from KIRC than the level of expression observed in healthy people $\left({ }^{*} P<0.05\right)$.

\section{MiR200c3p inhibits the migration of KIRC cells by targeting VEGFA}

VEGFA not only plays an important role in tumor angiogenesis but also plays an important role in tumor metastasis and growth. We observed that the mRNA level of VEGFA was up-regulated in the KIRC cells, particularly in the 786-0 and Caki-1 cell lines $(P<0.05$, Fig. 4A). Compared to other cell lines, 786-0 and Caki- 1 cells contained more amounts of VEGFA proteins $(P<0.05$, Fig. 4B). We conducted a DualLuciferase reporter assay. The results suggested that miR-200c-3p could directly target VEGFA.

The overexpression of miR-200c-3p can significantly reduce the luciferase activities in the 786-0 and Caki-1 cells (Figs. 4D and E). Results obtained using the qRT-PCR technique indicated that the level of VEGFA mRNA expression was low in 786-0 and Caki-1 cells. Overexpression of miR-200c-3p was also observed. High expression of VEGFA was observed in 786-0 and Caki-1 cells transfected with the miR$200 c-3 p$ inhibitor. In addition, results obtained using the western blot technique revealed that miR-200c-3p negatively regulated the expression of VEGFA (Figure 4G). Analysis of the results revealed that the expression of VEGFA in KIRC cells was high. It was also inferred that miR-200c-3p inhibited the migration of the KIRC cells by targeting VEGFA. 


\section{Discussion}

It is still a challenge to treat metastatic KIRC. It has been observed that several factors, such as VEGF, HIF$1 \mathrm{a}$, and Ecadherin [19-20],could regulate the metastasis of RCC. Unfortunately, the mechanisms of regulation of these factors are unclear. MiRNAs and small non-coding RNAs (19 to 24 nucleotide long) significantly influence the process of mRNA degradation and transformation inhibition. These are members of the class of non-coding RNAs that influence the development and progression of RCC by targeting key genes (HIF, mTOR, VEGF, and VHL).

We identified a hitherto unreported mechanism following which miR-200c-3p can inhibit KIRC migration and invasion by targeting VEGFA (Fig. 7). We used the bioinformatics tools and analyzed the GEO database for our studies. Low expression of miR-200c-3p was observed in KIRC tissue samples. Analysis of the data obtained using the qRT-PCR technique revealed that the level of miR-200c-3p expression in KIRC cells and tissue samples was significantly lower than the level of expression observed in normal renal cells. It was also observed that the overexpression of miR-200c-3p significantly suppressed cell migration and invasion in the Caki- 1 and 786-0 cells. The increased extent of cell migration and invasion was observed under conditions of miR-200c-3p knockdown. The migration rates observed in cells containing overexpressed miR-200c-3p were significantly lower than the migration rate recorded for the control group ( $P<0.01 ;$ Figs. $2 \mathrm{~B}, \mathrm{D}, \mathrm{E})$. These results agreed well with the results obtained by conducting the wound healing assay. Results from the wound healing assay revealed that the overexpression of miR$200 c-3 p$ resulted in reduced migratory abilities in Caki-1 and 786-0 cells. It was also observed that the inhibition of miR-200c-3p accelerated the process of wound closure. The results revealed that miR-200c$3 p$ could effectively inhibit the migration of the KIRC cells and the process of invasion. We determined the luciferase activities and conducted the western blot analysis to validate that VEGFA was a direct target of mi-200c-3p.

VEGF (including VEGF-A, VEGF-B, VEGF-C, VEGF-D, and VEGF-E) is secreted by various cells, including cancer cells. It is a member of the dimer glycoprotein family.And it promotes angiogenesis and helps in regulating the proliferation, invasion, and migration of endothelial cells [21]. The process of angiogenesis promotes the supply of nutrients to tumor cells. Angiogenesis also promotes the proliferation and development of tumor cells. Angiogenesis blockers help in cancer treatment. Treatment methods that target VEGF and/or VEGF receptors have been developed [13]. For example, bevacizumab, a monoclonal antibody that interrupts the interaction between VEGFA and its receptors (VEGFR1 and VEGFR2), has already been used in a clinical setting [22]. We observed that the expression of the VEGFA mRNA was low in KIRC cells with miR-200c-3p. On the contrary, high expression of VEGFA was observed in KIRC cells transfected with anti-miR-200c-3p. Furthermore, results obtained using the western blot technique suggested that miR-200c-3p negatively regulated the expression of VEGFA (Fig. 4G). The results revealed that miR200c3p inhibited the migration and invasion of the KIRC cells by targeting VEGFA (in vitro).

Thus, the downregulation of miR-200c-3p in KIRC helped suppress the proliferation, invasion, and migration of KIRC cells. Analyses of molecular mechanisms revealed that miR-200c-5p functioned as a 
tumor suppressor in KIRC by targeting VEGFA. Thus, it can be a potential treatment target for KIRC.

\section{Declarations}

\section{Acknowledgements}

Not applicable.

\section{Authors' contribution}

Yanmin Li, Hui Xu and Gang Xu designed and conducted the experiments and acquired data. Qianghai Wen and Xiaoqi Yan contributed to conception an designed the study. Yanmin Li, Hui Xu and Gang Xu drafted the manuscrip. All the authors contributed to edite the manuscript.

\section{Availability of data and materials}

All datasets generated for this study are included in the article material.

\section{Ethics approval and consent to participate}

The Ethics Committee of the First Affiliated Hospital of Gannan Medical University (Ganzhou, China) approved our study. The experiments in this study were conducted in accordance with the approved guidelines and norms. All the patients enrolled signed informed consent forms.

\section{Consent for publication}

Not applicable.

\section{Competing interests}

The authors declare that they have no conflict or financial interests.

\section{References}

1. Liu HT, Fan WX. Mirna-1246 suppresses the proliferation and migration of enal cell carcinoma through targeting cxcr4. Eur Rev Med Pharmacol Sci. 2020;24:5979-5987.

2. Siegel RL, Miller KD, Jemal A. Cancer statistics, 2016. CA Cancer J Clin. 2016 ;66:7-30.

3. Cheville JC, Lohse CM, Zincke H, Weaver AL, Blute ML. Compaisons of outcome and prognostic features among histologic subtypes of renal cell carcinoma. Am J Surg Pathol. 2003;27:612-624.

4. Ljungberg B, Campbell SC, Choi HY, Jacqmin D, Lee JE, Weikert S, et al. Theepidemiology of renal cell carcinoma. Eur Urol. 2011;60:615-621.

5. Quévillon Huberdeau M, Simard MJ. A guide to microrna-mediated gene silencing. Febs j. 2019;286:642-652. 
6. Cheng CJ, Bahal R, Babar IA, Pincus Z, Barrera F, Liu C, et al. Micrornasilencing for cancer therapy targeted to the tumour microenvironment. Nature. 2015;518:107-110.

7. Martello G, Rosato A, Ferrari F, Manfrin A, Cordenonsi M, Dupont S et al. A microrna targeting dicer for metastasis control. Cell. 2010;141:1195-1207.

8. Rupaimoole R, Slack FJ. Microrna therapeutics: Towards a new ea for the management of cancer and other diseases. Nat Rev Drug Discov. 2017;16:203-222.

9. Yang JS, Lai EC. Alternative mirna biogenesis pathways and the interprtation of core mirna pathway mutants. Mol Cell. 2011;43:892-903.

10. Hausser J, Zavolan M. Identification and consequences of mina-target interactions-beyond repression of gene expression. Nat Rev Genet. 2014;15:599-612.

11. Karimi Dermani F, Amini R, Saidijam M, Najafi R. Mir-200c, a tumor uppressor that modulate the expression of cancer stem cells markers and epithelial-mesenchymal transition in colorectal cancer. J Cell Biochem. 2018;119:6288-6295.

12. Liu J, Wang L, Li X. Hmgb3 promotes the proliferation and metstasis of glioblastoma and is negatively regulated by mir-200b-3p and mir-200c-3p. Cell Biochem Funct. 2018;36:357-365.

13. Cuzziol $\mathrm{Cl}$, Castanhole-Nunes MMU, Pavarino É C, Goloni-Betollo EM. Micrornas as regulators of vegfa and nfe2l2 in cancer. Gene. 2020;759:144994.

14. Zhang X, Tang J, Zhi X, Xie K, Wang W, Li Z, et al. Correctio: Mir-874 functions as a tumor suppressor by inhibiting angiogenesis through stat3/vegf-a pathway in gastric cancer. Oncotarget. 2017;8:29535.

15. English WR, Lunt SJ, Fisher M, Lefley DV, Dhingra M, Lee C, et al. Differential expression of vegfa isoforms regulates metastasis and response to anti-vegfa therapy in sarcoma. Cancer Res. 2017;77:2633-2646.

16. Liang H, Xiao J, Zhou Z, Wu J, Ge F, Li Z, et al. Hypoxia inducs mir-153 through the ire1 a-xbp1 pathway to fine tune the hif1a/vegfa axis in breast cancer angiogenesis. Oncogene. 2018;37:19611975.

17. Allen-Rhoades W, Kurenbekova L, Satterfield L, Parikh N, Fuja D, Shck RL, et Cross-species identification of a plasma microrna signature for detection, therapeutic monitoring, and prognosis in osteosarcoma. Cancer Med. 2015;4:977-988.

18. Marchionni L, Netto GJ, Hoque MO, Argani P. Micrornas, promising iomarkers in the diagnosis of xp11 translocation rcc-reply. Hum Pathol. 2017;68:206-207.

19. Maher ER. Genomics and epigenomics of renal cell carcinoma. Semin Cancer Biol. (2013) 23:10-17.

20. Chow TF, Mankaruos M, Scorilas A, Youssef Y, Girgis A, Mossad S, $t$ al. The mir-17-92 cluster is over expressed in and has an oncogenic effect on renal cell carcinoma. J Urol. 2010;183:743-751.

21. Fan H, Jin X, Liao C, Qiao L, Zhao W. Microrna-301b-3p accelerates he growth of gastric cancer cells by targeting zinc finger and btb domain containing 4. Pathol Res Pract. 2019;215:152667. 
22. Ferrara N. Vascular endothelial growth factor: Basic science and clinical progress. Endocr Rev. 2004;25:581-611.

\section{Figures}

A

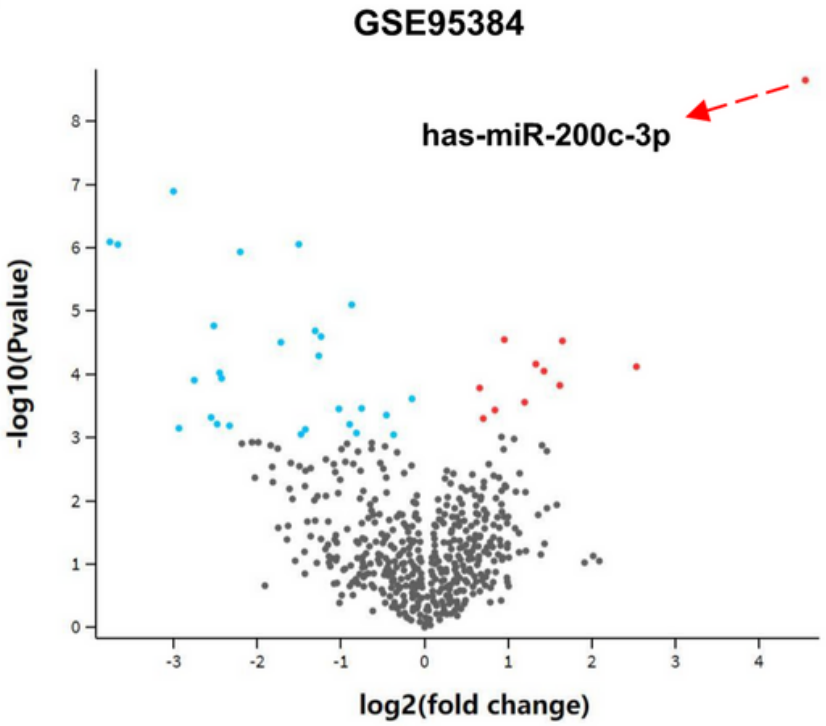

C

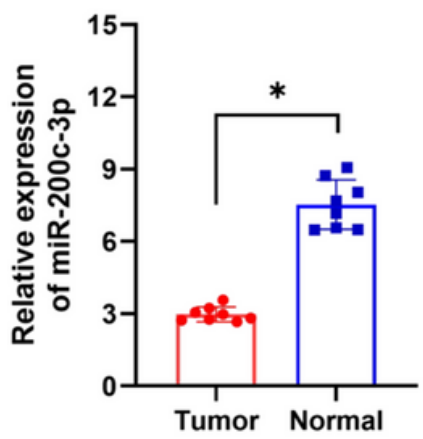

B

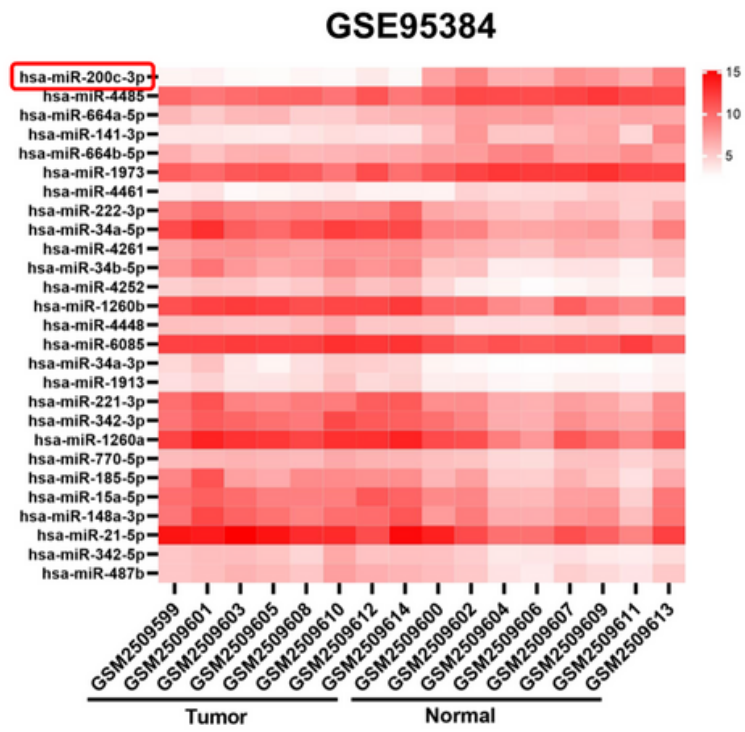

E

\section{Figure 1}

MiR-200c-3p is downregulated in KIRC tissues and cells. (A) Volcano plots indicating the differentially expressed miRNAs in KIRC tissues and adjacent tissues using data from GSE95384. (B) Heap mapping plots showing significantly different miRNAs. The color white indicates down-regulation and the color red indicates up-regulation in GSE95384. (C) MiR-200c-3p levels in KIRC tissues (based on the GSE95384 database). (D) QRT-PCR technique was used to determine the relative miR-200c-3p levels in 32 paired KIRC tissues and matched adjacent normal tissues. (E) MiR-200c-3p levels across HK-2 and different KIRC cell lines. Compared with Control group, *P0.05. 
A

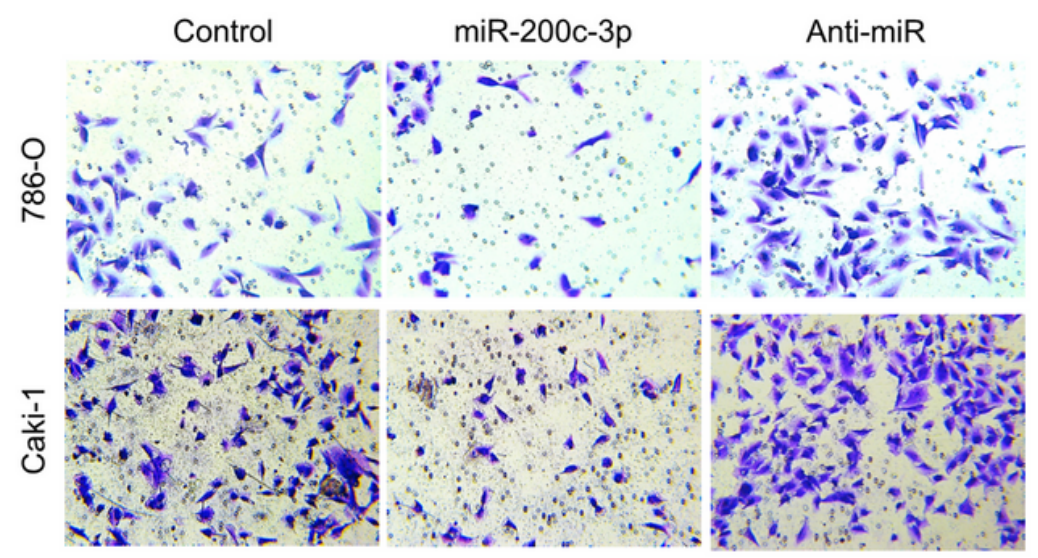

C

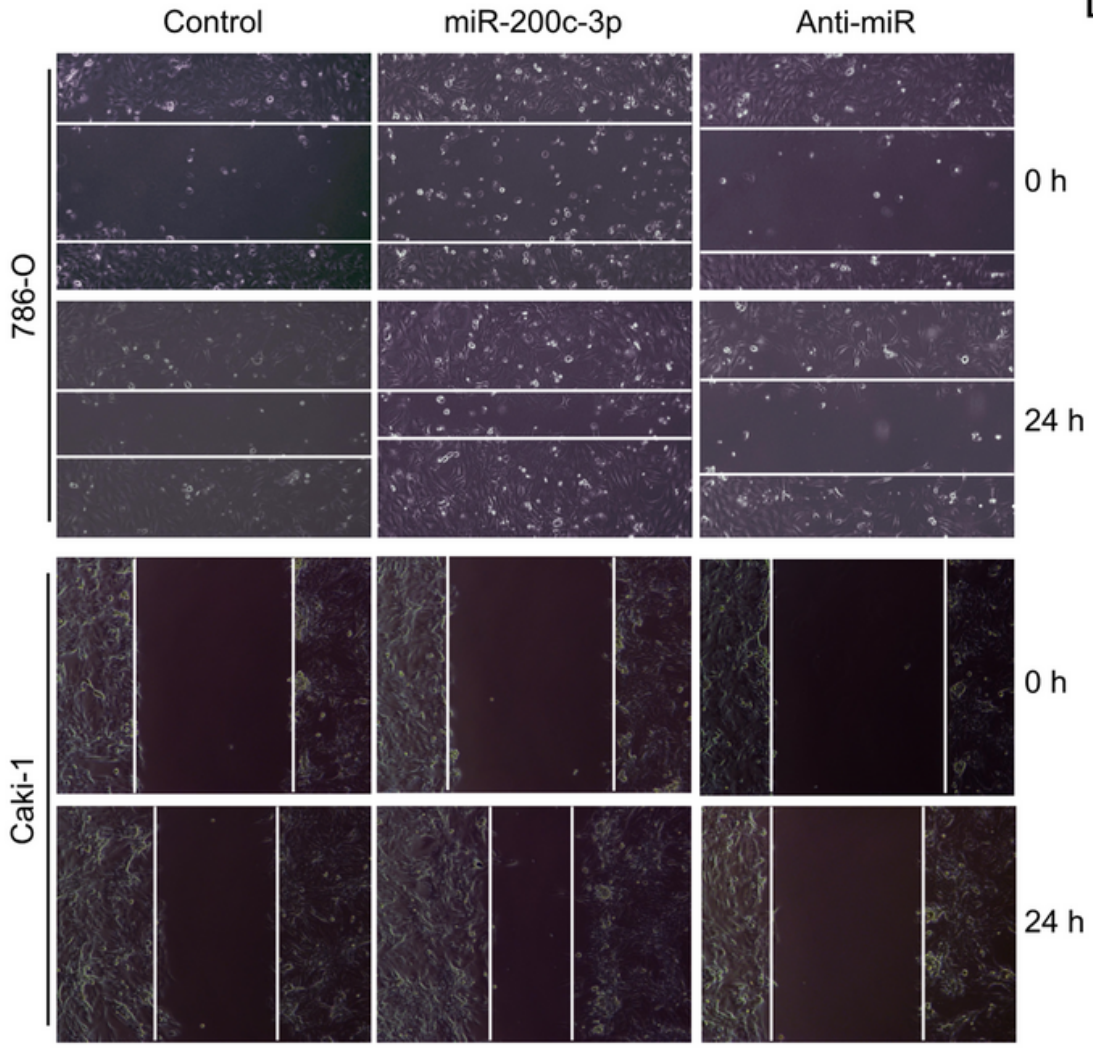

B

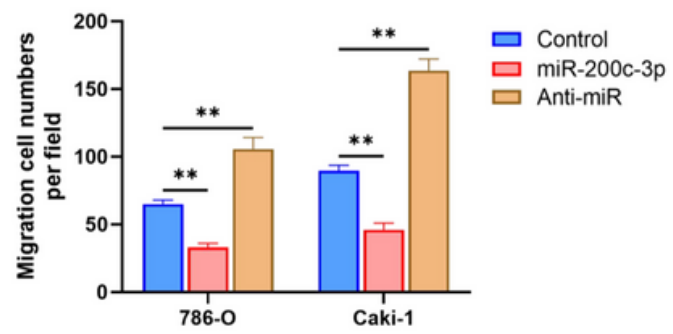

D

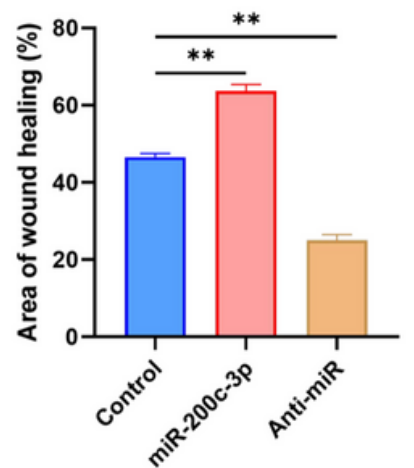

E

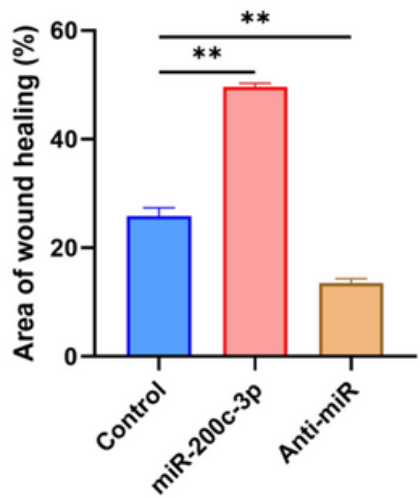

Figure 2

miR-200c-3p suppresses KIRC cells migration and EMT. Downregulation of miR-200c-3p promoted the migration of 786-O and Caki-1 cell lines in vitro. (A-E) Migration assay and wound healing assay were used to evaluate the migratory and invasive abilities of transfected 786-0 and Caki-1 cells $(n=3)$. The edges of the wounds are marked by white lines $(n=3)$. Compared with Control group, ${ }^{*} P<0.05$. ${ }^{*} P<0.01$. 
A

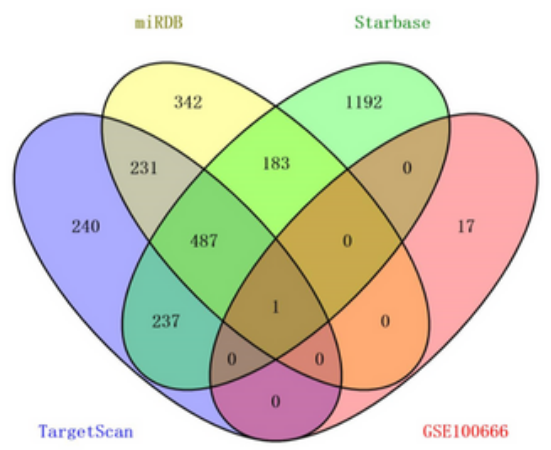

D
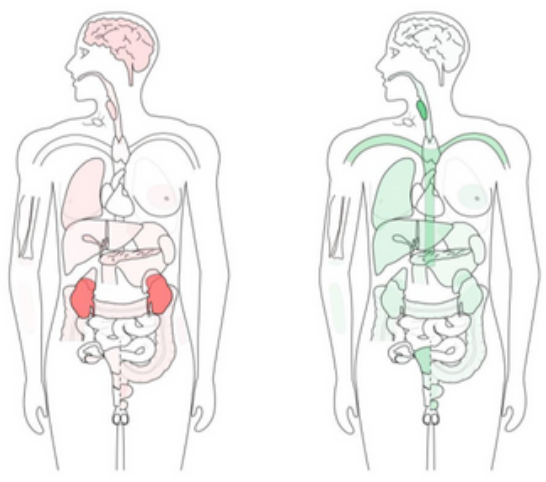

$\mathrm{F}$

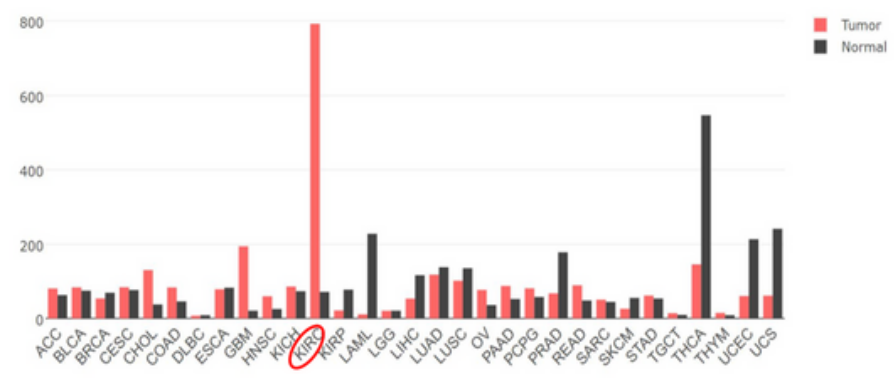

B

$\mathrm{E}$
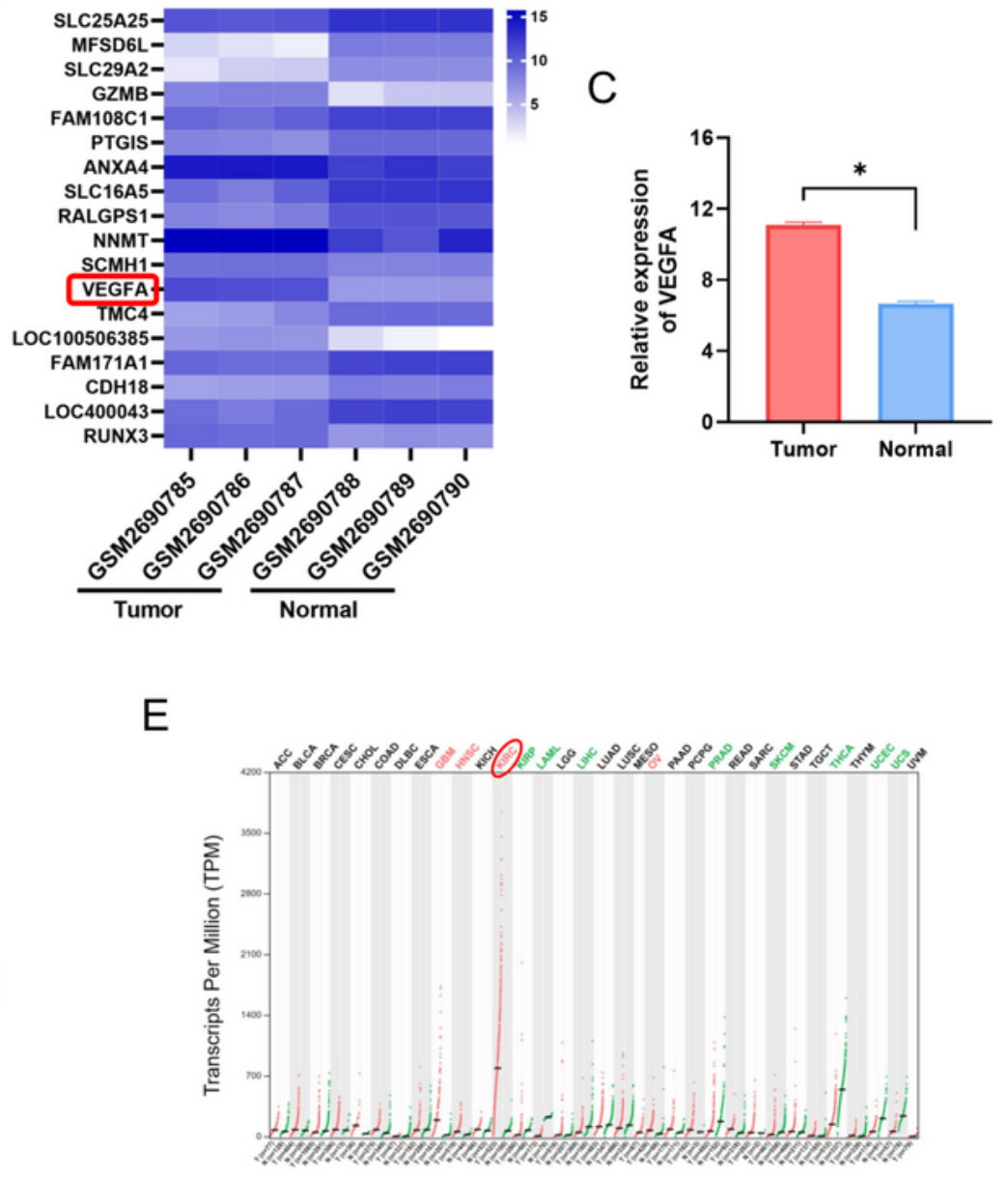

G

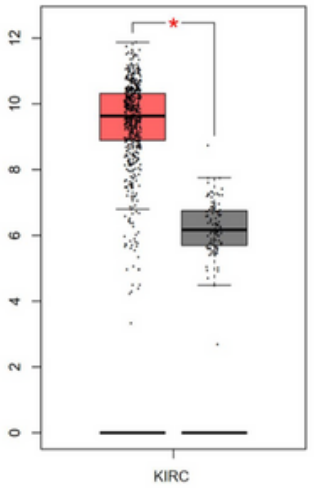

\section{Figure 3}

Identifying miR-200c-3p targets. (A) Overlapped target mRNAs were analyzed by miRDB, tarbase, targetScan, and GSE100666. (B) Expression of the 18 selected target genes was analyzed by constructing a heatmap. (C) VEGFA levels in the KIRC tissues of patients (based on the GSE100666 database). The VEGFA expression in adjacent tissues and KIRC tissues was determined using the qRTPCR technique. (D-G) VEGFA was analyzed using the data obtained from the GEPIA website. VEGF-A 
median expression in tumor-containing and normal samples in bodymap. The color red indicates tumor tissues and the color green indicates normal tissues. The level of expression of VEGFA was significantly higher in patients suffering from KIRC than the level of expression observed in healthy people $\left({ }^{*} P<0.05\right)$. The color red indicates tumor tissues and the color grey indicates norma tissues.

A

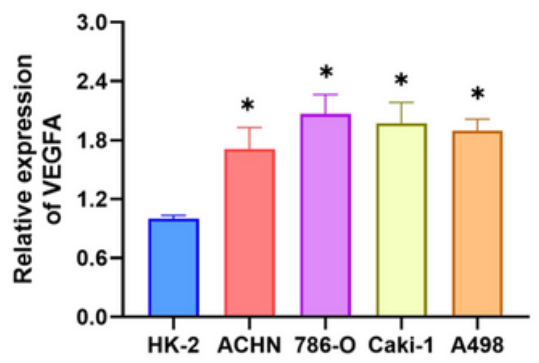

B

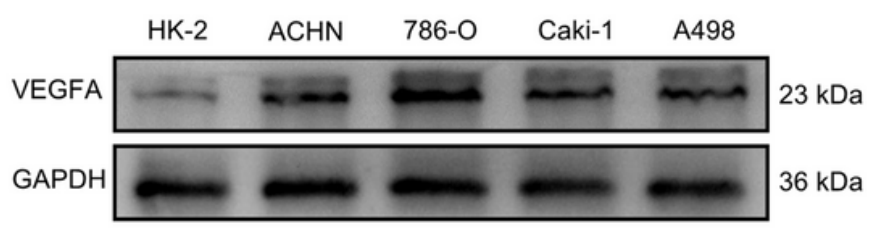

C Postion 1314-1320 of VEGFA 3'UTR WT 5'...AGUAGGGUUUUUUUCAGUAUUC...3' miR-200c-3p 3'...AGGUAGUAAUGGGCCGUCAUAAU...5'

Postion 1314-1320 of VEGFA 3'UTR MUT $\quad$ 5'...AGUAGGGUUUUUUUAUCAUAAC...3'

D

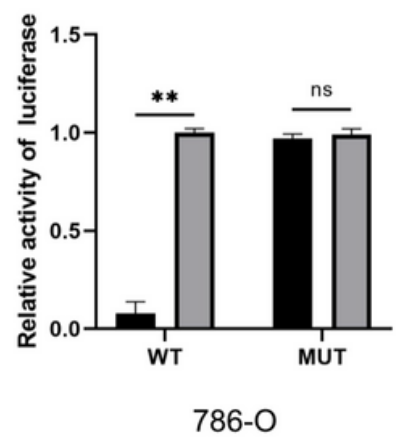

$\mathrm{F}$

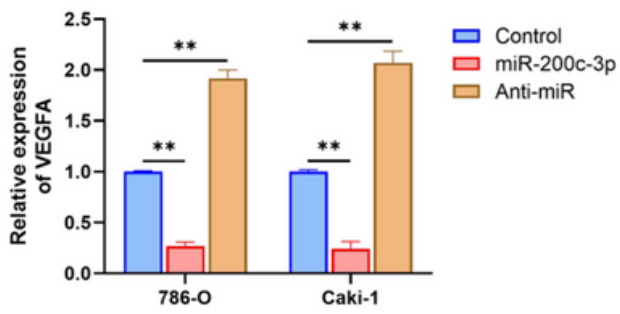

E

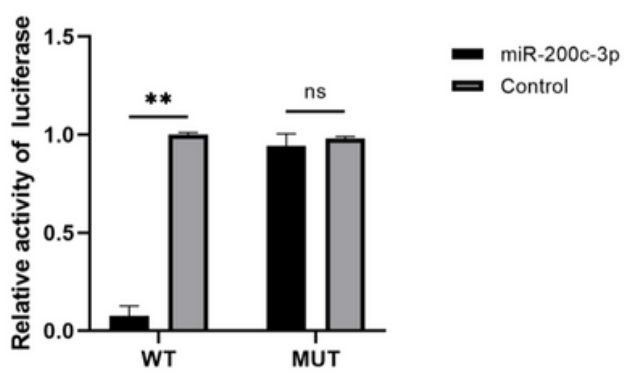

Caki-1

G

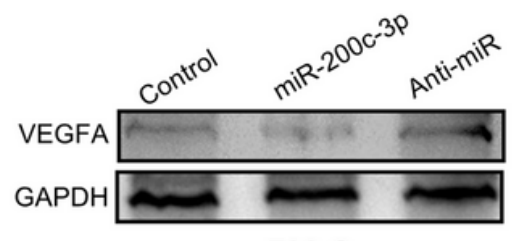

$786-0$

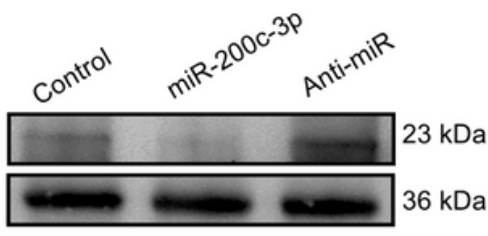

Caki-1

\section{Figure 4}

VEGFA is a direct target of miR 200c 3p. (A) VEGFA levels in HK-2 and KIRC cells. ${ }^{*} P<0.05$ vs. HK-2 ( $\left.n=3\right)$. (B) Protein level of VEGFA in different cell lines. (C) Predicted binding positions in VEGFA and miR-200c$3 p$ and the mutant sites on VEGFA. (D-E) Luciferase activities in 786-O and Caki-1 cells co-transfected with miR-200c-3p mimics or NC. (F) Results obtained using the qRT-PCR technique indicated that the VEGFA mRNA level was negatively regulated by miR-200c-3p in 786-0 and Caki-1 cells $(n=3)$. (G) Results 
obtained from the western blot tests confirmed that miR-200c-3p negatively controls VEGFA expression ( $n$ = 3). Compared with Control group, ${ }^{*}<<0.05$. 